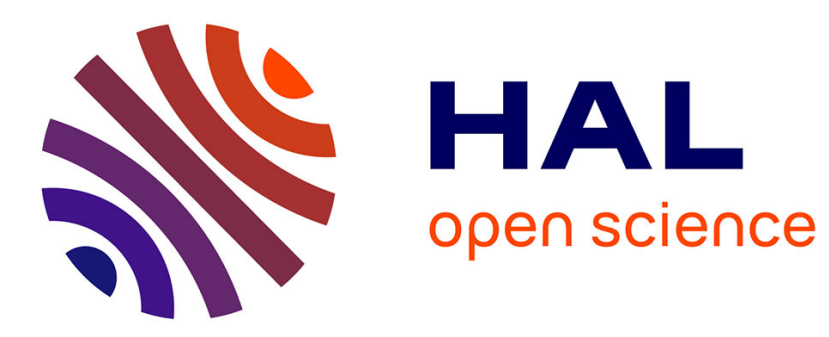

\title{
Optimal dynamics of soft shapes in shallow waters
}

Bijan Mohammadi, Afaf Bouharguane

\section{To cite this version:}

Bijan Mohammadi, Afaf Bouharguane. Optimal dynamics of soft shapes in shallow waters. Computers and Fluids, 2011, 40 (1), pp.291-298. 10.1016/j.compfluid.2010.09.031 . hal-00789951

\section{HAL Id: hal-00789951 \\ https://hal.science/hal-00789951}

Submitted on 19 Feb 2013

HAL is a multi-disciplinary open access archive for the deposit and dissemination of scientific research documents, whether they are published or not. The documents may come from teaching and research institutions in France or abroad, or from public or private research centers.
L'archive ouverte pluridisciplinaire HAL, est destinée au dépôt et à la diffusion de documents scientifiques de niveau recherche, publiés ou non, émanant des établissements d'enseignement et de recherche français ou étrangers, des laboratoires publics ou privés. 


\title{
Optimal dynamics of soft shapes in shallow
}

\author{
waters
}

\author{
Bijan Mohammadi and Afaf Bouharguane \\ Institut de Mathématiques et de Modélisation de Montpellier \\ Université Montpellier II, CC51, 34095 Montpellier, France \\ Published by Computers and Fluids, \\ DOI:10.1016/j.compfluid.2010.09.031
}

Abstract - A sandy sea bottom is seen as a structure with low stiffness which adapts to the motion of water in a shallow domain described by the Saint Venant equations. The coupling is based on the minimization of water wave energy with minimal sand transport. The approach is shown being similar to the use of an original Exner equation for the bottom with non local flux expressions. Also, examples of the applications of the framework to inverse problems in coastal engineering are shown.

Keywords: Bottom sea morphodynamics, level set, shape optimization, fluid-structure coupling, Saint Venant Equations, sensitivity analysis by adjoint, Exner equation.

\section{Introduction}

In coastal morphodynamics some events occur over small space and time scales. One can cite the removal of sediment from the toe of coastal structures which often occurs and recovers completely during the course of a single tide (hourly 
basis). Then, the time and space scales concern storm response lasting for a few tides (say daily basis). Here the beach can be modified both long and cross-shore by hundreds of meters. Recovery between storms will take longer (monthly basis). This classification can continue with seasonal and inter-annual variability. Hence, coastal morphodynamics occur over a broad range of time and length scales where the spatial scale increases with the timescale. This latter remark also implies that long-shore transport gains importance over cross-shore with increasing timescale.

The litterature on coastal morphodynamics use either local concepts such as sediment transport using fluid-induced shear in the sediment modelling and bottom friction coefficient or global ones such as global beach morphodynamics based on long and cross-shore fluxes and global beach lines dynamics $[7,8,19]$.

We would like to unify local and global analysis by the application of the control theory to the evolution of sandy bottom seas. This melange of local and global natures is made evident when looking at how the question of bottom morphodynamics is often treated in the literature using the solution of an Exner equation [25]. One will indeed show that the approach provides a new flux term for the Exner equation linking local and global informations.

In the past we have used minimization principle to design defence structures against beach erosion $[3,4]$. In these works, the designed structure were independent of time and were built once for all. Here, we would like to go one step further giving the possibility to the structure to change in time. One particular case is then the sea bottom seen as a structure with low stiffness. The fundamental assumption is that the bottom adapts to the flow by some sort of optimal sand transport in order to minimize some energy expression. Optimal transport can be seen as minimal change in the bottom shape. The approach is not limited by the particular expressions we consider here for the functional nor by the flow equations which can also be more sophisticated.

The paper starts with the problem of bottom motion formulated as an optimal control problem. Shallow water equations are briefly recalled with emphasis 
on boundary conditions by level set for emerged structures. Follows a discussion on the choice of cost functions. A comparison is then made between modelling the transport of sediments by the Exner equation and by our minimization principle. Sensitivity evaluation being an important issue in this calculus of variation problem, a model problem is introduced to discuss various questions on complexity and the need for the adjoint equation for the flow equation. The extension to the case of the Saint Venant equations is also presented. Throughout the paper, one shows simulations featuring qualitative behavior of the approach.

\section{Bottom motion as a control problem}

In what follows, the parameterization of the bottom sea and the corresponding bathymetry are both denoted by $\psi$. The latter is a two dimensional positive function $\psi(x, y): \Omega \subset \mathbb{R}^{2} \rightarrow \mathbb{R}^{+}$and the former is a multidimensional function in an admissible space $O_{a d} \subset \mathbb{R}^{n}$ with $n$ the dimension of the parameter space and defining uniquely $\psi(x, y)$.

We consider a control problem where the control is the bottom sea represented through a parameterization $\psi . \psi$ changes with time following the changes in the state given by the flow conditions $\mathbf{U}$. The admissible space is to specify not any shape can be taken by the bottom. For instance, $\psi$ should be a univocal function with some regularity. The regularity is specified through the parameterization chosen. We assume that $\psi$ changes in order to minimize a time dependent functional:

$$
\min _{\psi \in O_{a d}} J(\psi, \mathcal{U}(\psi)),
$$

where $\mathcal{U}(\psi)=\{\mathbf{U}(\psi, \tau), \tau \in[t-T, t]\}$ gathers the state evolution in time solution of some state equation (here the shallow water equations). The cost 
function involves this state evolution as, for instance, in:

$$
J(\psi, \mathcal{U}(\psi))=\int_{t-T}^{t} j(\psi, \mathbf{U}(\psi, \tau)) d \tau
$$

$T$ indicates a time dependency window and also permits to introduce a difference in time scales between the bottom sea and the flow motions. We will see examples of such functionals in section 4 .

\section{Governing equations for shallow waters}

Given a sea bed bathymetry $\psi$, the two-dimensional nonlinear shallow water or Saint Venant equations [5] with topobathymetry are:

$$
\partial_{t} \mathbf{U}+\partial_{x} F(\mathbf{U})+\partial_{y} G(\mathbf{U})=S(\mathbf{U})
$$

where $\mathbf{U}={ }^{t}(h, h u, h v), F(\mathbf{U})={ }^{t}\left(h u, h u^{2}+\frac{g}{2} h^{2}, h u v\right), G(\mathbf{U})={ }^{t}\left(h v, h u v, h v^{2}+\right.$ $\left.\frac{g}{2} h^{2}\right)$ and $S(\mathbf{U})={ }^{t}\left(0,-g h \partial_{x} \psi,-g h \partial_{y} \psi\right) . \quad \mathbf{u}={ }^{t}(u, v)$ the depth-averaged velocity with $u$ and $v$ the scalar components in the horizontal $x, y$ directions and $h$ is the local water depth. $\mathbf{U}$ is the vector for the conservative variables, $F(\mathbf{U})$ and $G(\mathbf{U})$ stand for the flux functions respectively along the $x$ and $y$ directions and $S(\mathbf{U})$ represents the bed slope source term.

These equations are discretized by a finite volume formulation [10]. Our finite volume implementation preserves steady state solutions on non flat bottom seas in the absence of perturbations $[1,2]$. It is also suitable to capture wetting and drying phenomena $[18,17]$.

Overall, four boundary conditions are needed at slip, inlet, shoreline and outlet boundaries. The slip boundary condition $(\mathbf{u} . \mathbf{n}=0)$ is naturally taken into account in a finite volume formulation. The outlet condition for open seas is a transmissive boundary condition. Values at boundary cells are obtained by second order extrapolations normal to the boundary from the values inside the domain. To describe incoming waves we use an absorbing/generating inlet 
boundary condition where the values of water depth are prescribed. To simulate sea conditions, water depth variations at inlet are obtained by addition of monochromatic waves following, for instance, a Jonswap energy spectral distribution [14, 13]. In subcritical regimes, characteristic curves and Riemann invariants provide normal velocity. In open sea we allow for reflected waves to freely exit the domain not creating reflection [16]. The shoreline is an implicit function of the flow and the bed. Dry cells are those where the water depth is below a given value (say $10^{-10}$ ). Once those identified, the shoreline location is solution of Riemann problems at the interfaces between wet and dry cells [18].

For the basin simulations presented here only the slip and inlet conditions have been applied as there is not outgoing wave in the basin and also water depth never vanishes here so none of the open sea or the shoreline conditions have been activated.

Time integration is explicit for the flow. Due to the difference of the time scales between fluid motion and changes in sea beds, several times steps will be taken in the flow solver before a new time step by the bed model.

\subsection{Level set}

When there is an emerged structure or bathymetry, slip boundary condition needs be applied along the emerged surface. In order to avoid fitting the mesh to the contour of emerged structures, we use their level set representation and do not modify the mesh to account for their presences. Level set is an established technique to represent fixed or moving interfaces on cartesian grids. Immersed boundary, fictitious domain methods as well as penalizing techniques are methods to impose boundary conditions on surfaces which are not unions of edges and faces of elements of the (non-body fitted) computational mesh [9, 24, 26, 23].

A parametrization of a boundary $\Gamma$ of a domain $\Omega$ by the level set method is based on the zero-level curve of a function $\phi$ :

$$
\Gamma=\{x \in \Omega: \phi(x)=0\}
$$


The function $\phi$ could be the signed Euclidean distance to $\Gamma$ :

$$
\phi(x)= \pm \inf _{y \in \Gamma}|x-y|
$$

with the convention of a plus sign if $x \in \Omega$ and minus sign otherwise. Hence

$$
\left.\phi\right|_{\Gamma}=0,\left.\quad \phi\right|_{\mathbb{R}^{2} \backslash \Omega}<0,\left.\quad \phi\right|_{\Omega}>0
$$

The definition can be extended to open shapes by using $\Gamma^{ \pm}$instead of $\Omega$.

For a given shape given by (4) the normal to $\Gamma$ is $n=\nabla \phi /|\nabla \phi|$ at $\phi=0$.

A relaxed characteristic function of $\Omega$ is

$$
\chi=\max \left(0, \phi /\left(|\phi|+\varepsilon_{o p t}(h)\right)\right)
$$

where $\varepsilon_{\text {opt }}(h)$ is a strictly positive relaxation function which tends to zero with the background mesh size $h$. It is defined solving minimization problems for a sampling in $h$ :

$$
\varepsilon_{o p t}(h)=\operatorname{argmin}_{\varepsilon(h)>0}\left\|u_{h}\left(\chi\left(\phi_{h}(\varepsilon(h))\right)\right)-\Pi_{h} u_{r e f}\right\|
$$

where $\Pi_{h}$ is the restriction operator to mesh $h$ and $u_{h}$ the discrete state. The numerical results given below have been computed with $\varepsilon_{\text {opt }}(h)=c h$ for some constant $c>0$. This choice guarantees the consistency of the scheme. The coefficient $c$ is fitted, once for all, in order to minimize the error $\left\|u_{r e f}-u_{h}^{\varepsilon}\right\|$ for a reference solution $u_{r e f}$ which can be either a solution obtained with a body fitted mesh or, when available, an analytical solution.

Once $\phi_{h}$ is known, we take into account the boundary conditions for a the shallow water equations through:

$$
\left(\partial_{t} \mathbf{U}_{h}+\partial_{x} F_{h}\left(\mathbf{U}_{h}\right)+\partial_{y} G_{h}\left(\mathbf{U}_{h}\right)-S_{h}\left(\mathbf{U}_{h}\right)\right) \chi\left(\phi_{h}\right)+\mathcal{E}_{h}\left(\mathbf{u}_{h} \cdot \mathbf{n}_{h}\right) \delta_{\phi_{h}}=0
$$

Here $\mathcal{E}_{h}$ builds an extension of the boundary condition on $\Gamma_{h}$ over the domain 
$\Omega_{h}$ and $\delta_{\phi}$ is a relaxed Dirac measure which is constructed using $\chi\left(\phi_{h}\right)$ and whose support approximates the boundary. In other words, the shallow water equations degrade to an algebraic equation on the velocity when $\chi$ tends to zero. At this level, we have still a limitation: the structure can be either submerged or fully emerged. One does not target situations where the water pass and runs over the structure and drops afterward. This is not a limitation for our problem as these situations do not represent much in term of water wave energy (see section 4$)$.

Figure 1 shows an example of an incoming wave where slip boundary condition is enforced along a cylindrical structure using (6).

\section{Cost function}

One of the main ingredients in the chain of control is the cost function to be minimized. One expects the bottom to act as a flexible structure and to adapt to flow conditions in order to minimize some energy-based functional. One can, for instance, consider an energy made of the sum of instantaneous water mechanical energy and involving a constraint on sand displacements requiring minimal bathymetry changes from $\psi(t-T)$ at the beginning of a time interval of influence $T$. We assume that the influence of water conditions on a sandy bottom at a given instant does not involve time history of more than a few wave periods:

$$
J_{1}(\psi)=\int_{t-T}^{t} \int_{\Omega}\left(\frac{1}{2} \rho_{w} g \eta^{2}+\rho_{s} g(\psi(\tau)-\psi(t-T))^{2}\right) d \tau d \Omega
$$

where $\Omega$ is the physical domain, $\rho_{w}$ and $\rho_{s}$ respectively the water and sand density and $\eta$ is the flow elevation defined as:

$$
\eta(x, y, \psi, t)=h(x, y, \psi, t)-\frac{1}{T} \int_{t-T}^{t} h(x, y, \psi, \tau) d \tau
$$


This functional aims at reducing wave elevations with minimal changes in the original bottom. Minimizing $J_{1}$ does not mean that a flat bottom will remain flat. Indeed, one observes the apparition of ripples and dunes on a flat bottom even at moderate flow speeds.

We have observed that controlling water wave energy is crucial to fight erosion. Indeed, this is responsible for sediments put in suspension by generating bottom orbital velocity $[19,8,4]$. Figure 2 shows an example of bottom sea changes due to interactions with water motion based on the minimization of (7) after about one day. The approach predicts the apparition of two longshore natural sand bars. The bars appear after six hours and remain stable in time. Water elevation in time is prescribed on the left boundary which creates waves entering normal to this boundary. The starting bottom profile is linear. The apparition of sand bars is compatible with what observed in nature.

A fundamental idea motivating the introduction of the second term in the functional (7) is the concept of optimal transport. In other words, one thinks that the bottom will adapt to the flow in some optimal way in term of sand transport. A more general functional could therefore involve the Wasserstein distance between two bottom seas. Suppose $\psi(t-T, x, y)$ and $\psi(t, x, y)$ are positive function describing two bathymetries at instants $t-T$ and $t$ such that the total amount of sand is conserved:

$$
\int_{\Omega} \psi(t, x, y) d \Omega=\int_{\Omega} \psi(t-T, x, y) d \Omega
$$

An optimal transport of $\psi(t-T, x, y)$ to $\psi(t, x, y)$ will consist in finding the application $M: \Omega \rightarrow \Omega$ realizing the transformation from $\psi(t-T, x, y)$ to $\psi(t, x, y)$ minimizing:

$$
d_{W}(\psi(t-T, x, y), \psi(t, x, y))=\int_{\Omega}\|(x, y)-M(x, y)\|^{2} \psi(t-T, x, y) d \Omega
$$


and such that for all continuous function $\varphi$

$$
\int_{\Omega} \psi(t-T, x, y) \varphi(M(x, y)) d \Omega=\int_{\Omega} \psi(t, x, y) \varphi(x, y) d \Omega
$$

$d_{W}$ is a measure of the transport effort from one bathymetry to another. One knows that there is a unique $M=\nabla \phi$ with $\phi$ a convex potential satisfying (9) $[27,6,21]$. And, as the bathymetries in sandy bottom seas are strictly positive and continuous functions then one can look for $\phi$ solution of the Monge-Ampère Equation (MAE) $(H(\phi)$ being the Hessian of $\phi$ and $\operatorname{det}(H)$ its determinant),

$$
\operatorname{det}(H(\phi(x, y)))=\frac{\psi(t-T, x, y)}{\psi(t, \nabla \phi(x, y))}
$$

In other words, the governing equations for our fluid-structure system are the Shallow water equations for the fluid, and the Monge-Ampère equation for the structure and one would like to minimize the total water mechanical energy under these PDE constraints. Solving the MAE equation is a difficult task and we would like to avoid it. The second term in cost function (7) is an upper bound for $d_{W}$.

The previous functional involves water elevations. In the same way, one could imagine that bottom sea will adapt to the flow in order to reduce its horizontal velocity gradients with minimum variation in bathymetry. A second functional can then be built:

$$
J_{2}(\psi)=\int_{t-T}^{t} \int_{\Omega}\left(\left\|\nabla_{x y} \mathbf{u}\right\|+\rho_{s} g(\psi(\tau)-\psi(t-T))^{2}\right) d \tau d \Omega
$$

where $\nabla_{x y}$ indicates the spatial gradient of the variables along $x$ and $y$ and the norm stands for $\left\|\nabla_{x y} \mathbf{u}\right\|=\left(u_{x}^{2}+v_{x}^{2}+u_{y}^{2}+v_{y}^{2}\right)^{1 / 2}$. Figure 3 shows an example of bathymetry changes after 20 hours starting from the same linear initial bottom profile than in Figure 2 and applying the same water elevation in time on the left boundary. One notices a transfer from cross-shore to long-shore variations. The latter was zero initially. The cost function is reduced during 
time as the bottom adapts to the flow. Figure 4 shows the same analysis but with an emerged cylindrical structure represented by the level set technique. The cost function reduction is less efficient as some part of the bottom is now rigid and the emerged structure amplifies the flow gradients.

Another cost function of interest involves average current patterns:

$$
J_{3}(\psi)=\left(\frac{1}{T} \int_{\Omega}\left(\int_{0}^{T} \mathbf{u} d \tau\right)-\mathbf{u}_{\mathbf{d e s}} d \Omega\right)^{2},
$$

where $\mathbf{u}_{\mathbf{d e s}}$ indicates a target mean current distribution. Minimizing this functional means finding the bottom sea shape which realizes 'best' a mean flow pattern. Of course, as often in inverse problems, existence of a solution is not obvious. Indeed, it is not always possible to find a shape realizing the required state distribution.

Figure 5 shows an example of application. In a square basin of side $L$, we start from a flat bottom for which the mean current vanishes for periodic flow elevations prescribed on the left boundary. we aim at finding a new shape realizing a given target mean flow current. Here, the target mean current is given by $\frac{1}{T} \int_{0}^{T} \mathbf{u} d \tau=(a, 0)$ for $y \in[0, L / 3] \cup[2 L / 3, L]$ and $(-2 a, 0)$ for $y \in$ $[L / 3,2 L / 3]$. One sees that a non intuitive bottom shape found by minimization almost realizes the target mean current. Once this is achieved, the functional and gradient are small and the bottom shape does not change anymore. Here the bottom shape does not depend on time because the target mean current is taken stationary. One could have targeted a mean current with low frequency time variations. Then the bottom shape adapts to the variation in time of $\mathbf{u}_{\mathrm{des}}(t)$ and the cost function is then time dependent too.

$$
J_{4}(t, \psi)=\left(\frac{1}{T} \int_{\Omega}\left(\int_{t-T}^{t} \mathbf{u} d \tau\right)-\mathbf{u}_{\mathbf{d e s}}(t) d \Omega\right)^{2},
$$




\section{Minimization principle and the Exner equation}

Consider the following equation which in discrete form minimizes $J(\psi)$ :

$$
\psi_{t}=-\rho \nabla_{\psi} J, \quad \psi(t=0, x, y)=\psi_{0}
$$

Here $\rho$ characterizes the ability of sand to be put in motion by water. Together with the interval of influence $T$, mentioned in (7), $\rho$ is the second parameter to be assimilated using experimental data.

One well-known approach to model bottom motion is through the Exner equation [25]. This equation models the conservation of mass between in the bed and transported sediments. More precisely, this is a conservation equation for the mass of sediments in the bed of a channel and sediments that are in motion due to transport by the flow. Bed elevation or degradation are supposed linear with respect to the amount of sediment that drops out or becomes entrained by the flow:

$$
\psi_{t}+\frac{1}{1-\lambda_{p}} \nabla \cdot q=0, \quad \psi(t=0, x, y)=\text { given }
$$

where $\lambda_{p} \in\left[0,1\left[\right.\right.$ is the porosity of the bed. One remarks that $1 /\left(1-\lambda_{p}\right)$ plays the role of $\rho$ in the minimization equation (15). This increases with the porosity of the bed. To link both analysis we define $\rho$ by $\rho=1 /\left(1-\lambda_{p}\right)^{n}, 1 \leq n . q$ is usually a function of $\mathbf{U}$ and involves several constants characterizing the sand. Lack of data is one major difficulty with this approach. This is why Exner equation is often considered in one dimension in space

$$
\psi_{t}+\frac{1}{1-\lambda_{p}} q_{x}=0, \quad \psi(t=0, x)=\text { given }
$$

where one simple and popular expression for $q$ is of the form $q \sim u|u|^{m-1}$ with $m>1[28]$. 
Equation (15) is an Exner equation with a non local flux:

$$
q(x)=q(-\infty)+\int_{-\infty}^{x}\left(1-\lambda_{p}(\zeta)\right)^{1-n} J_{\psi}(\zeta) d \zeta
$$

where one can suppose that $q(-\infty)=0$ as $x \rightarrow-\infty$ denotes offshore locations and far from the shallow domain where the effect of the flow motion on the bottom sea is negligible. In the same way, $J_{\psi}(\zeta) \rightarrow 0$ when $\zeta \rightarrow-\infty$. This also means that the integral will have a finite support. One can also notice that the main contributions to the flux come from regions in the bed where $\lambda_{p} \rightarrow 1$ (porous).

Now, consider $J=\frac{1}{2} u_{x}^{2}$. Minimizing $J$ would force the bottom to adapt in order to reduce gradients in the flow. This is similar to what we expect minimizing (12). For this functional $q$ reads:

$$
q(x)=\int_{-\infty}^{x}\left(1-\lambda_{p}\right)^{1-n} u_{\zeta} u_{\psi \zeta} d \zeta
$$

and integrating by part,

$$
q(x)=-\int_{-\infty}^{x}\left(\left(1-\lambda_{p}\right)^{1-n} u_{\zeta}\right)_{\zeta} u_{\psi} d \zeta+\left(1-\lambda_{p}(x)\right)^{1-n} u_{x}(x) u_{\psi}(x) .
$$

This shows a local term plus a global correction involving second order derivatives of the state upstream. Similar nonlocal terms can be found, for instance, in a model by Fowler for the motion of sand dunes [11].

\section{Sensitivity evaluation}

Sensitivity evaluation for large dimension minimization problems, like our situation, is not an easy task. The most efficient approach is to use an adjoint variable with the difficulty that it requires the development of a specific software. Automatic differentiation (AD) brings some simplification, but does not avoid the main difficulty of intermediate states storage in time dependent calcu- 
lations. Check-pointing technics bring, however, some relief [12]. In this section, we explain the adjoint method through a model problem and its computer-based implementation through automatic differentiation for a time-dependent model problem [20, 22].

\subsection{A model problem}

Consider the following time dependent state equation for $u(y, t), \quad-\psi \leq y \leq$ $\psi, t \geq 0$ in a infinite channel of width $2 \psi$.

$$
u_{t}-u_{y y}=F(\psi, y, t), \quad u(\psi, t)=u(-\psi, t)=0,
$$

with

$$
F(\psi, y, t)=-\varepsilon \omega \sin (\omega t)\left(\psi^{2}-y^{2}\right)+2(1+\varepsilon \cos (\omega t)),
$$

inducing small perturbation in time around a parabolic solution if $\varepsilon<<1$. The exact solution for this equation is:

$$
u(y, t)=\left(\psi^{2}-y^{2}\right) f(t), \quad f(t)=(1+\varepsilon \cos (\omega t)) .
$$

And consider a functional $J$ of the form:

$$
J(\psi, t)=\psi^{m} u_{y}(y=\psi, t), \quad m \in \mathbb{N}^{*}
$$

involving instantaneous state quantities. The sensitivity with respect to $\psi$ is:

$$
J_{\psi}(\psi, t)=m \psi^{m-1} u_{y}(\psi, t)+\psi^{m} u_{y_{\psi}}(\psi, t) .
$$

The first term is what we call incomplete sensitivity [20] where the sensitivity of the state with respect to the shape is neglected. This is a very strong approximation. But, as we have:

$$
u_{y}(\psi, t)=-2 \psi f(t), \quad \text { and } \quad u_{y_{\psi}}(\psi, t)=-2 f(t),
$$


the different contributions in (19) can be expressed:

$$
J_{\psi}(\psi, t)=m \psi^{m-1}(-2 \psi f(t))+\psi^{m}(-2 f(t)) .
$$

Comparing with $-2(m+1) \psi^{m} f(t)$, one sees that the incomplete sensitivity approximation of the gradient is accurate and its precision increases with $m$. Most important, the incomplete sensitivity has always the right sign. It is obvious that the analysis still holds if the functional involves a time integral:

$$
J(\psi, T)=\int_{(0, T)} \psi^{m} u_{y}(y=\psi, t) d t
$$

Now, if the functional involves an integral over time and space:

$$
J(\psi, T)=\int_{(0, T) \times(-\psi, \psi)} j(y, t) d t d y, \quad j(\psi, t)=\psi^{m} u_{y}(y=\psi, t), \quad m \in \mathbb{N}^{*}
$$

Linearizing $J$ one has:

$$
\begin{gathered}
J_{\psi}(\psi, T)=\int_{(0, T) \times(-\psi, \psi)}\left(m \psi^{m-1} u_{y}(\psi, t)+\psi^{m} u_{y_{\psi}}(y, t)\right) d t d y \\
+\int_{(0, T)}\left[\psi^{m} u_{y}(y, t)\right]_{ \pm \psi} d t .
\end{gathered}
$$

An incomplete evaluation of the sensitivity is still accurate because $u_{y_{\psi}}=0$. One also notices that if $m$ is odd the last integral vanishes. However, this integral is cheap to get as it does not involve any state sensitivity with respect to $\psi$.

Incomplete sensitivity is therefore efficient and very cheap to get because it does not require the linearization of the state equations. However, it only holds for functionals of the form:

$$
J(\psi, T)=\int_{(0, T)} j(\psi, t) d t
$$


where $j$ features a separation in variables $\psi$ and $u[20]$ :

$$
j(\psi, t)=\alpha(\psi) \beta(u),
$$

with $\alpha$ and $\beta$ differentiable functions.

Now, if $J$ is of the form:

$$
J(\psi, T)=\int_{(0, T) \times(-\psi, \psi)} j(y, t) d t d y
$$

with $j$ arbitrary, the sensitivity reads:

$$
J_{\psi}(\psi, T)=\int_{(0, T) \times(-\psi, \psi)}\left(j_{\psi}+j_{u} u_{\psi}\right) d t d y+\int_{(0, T)} j( \pm \psi, t) d t .
$$

In this expression only $u_{\psi}$ is costly to get as it requires the linearization of the state equation.

The solution of the linearized state equation (17) permits to write for all function $v$ :

$$
0=\int_{(0, T) \times(-\psi, \psi)}\left(\left(u_{\psi}\right)_{t}-\left(u_{\psi}\right)_{y y}-F_{\psi}\right) v d t d y .
$$

Integrating by part, it gives:

$0=\int_{(0, T) \times(-\psi, \psi)}\left(-v_{t}-v_{y y}\right) u_{\psi} d t d y+\int_{(0, T) \times(-\psi, \psi)}-F_{\psi} v d t d y+\int_{(-\psi, \psi)}\left[v u_{\psi}\right]_{T}^{0} d y$.

Let us introduce a backward adjoint problem, suitable when the dimension of the control space parameter is large (as it is the case in our problem of bottom sea modification):

$$
v_{t}-v_{y y}=j_{u}, \quad v(y, T)=v( \pm \psi, t)=0 .
$$

Therefore, with $v$ solution of the backward adjoint equation (22) with the chosen 
boundary and final conditions one has:

$$
\int_{(0, T) \times(-\psi, \psi)} j_{u} u_{\psi} d t d y=\int_{(-\psi, \psi)} u_{\psi}(0) v(0) d y-\int_{(0, T) \times(-\psi, \psi)} F_{\psi} v d t d y .
$$

The important point here is that, unlike with the linearized equation, with $\psi$ of any dimension $v$ is computed only once before assembling the right-hand-side above.

Here, the state equation is linear and no storage of intermediate states was necessary in adjoint calculation. On the other hand, if the state equation is nonlinear, solution of the adjoint equation requires the storage all intermediate states between 0 and $T$. For instance, consider

$$
u_{t}+u u_{y}-u_{y y}=F(t), \quad u(\psi, t)=u(-\psi, t)=0 .
$$

For the same functional (21), the adjoint equation in this case reads

$$
v_{t}+u v_{y}-v_{y y}=j_{u}, \quad v(y, T)=v( \pm \psi, t)=0,
$$

$u$ is now present in the left-hand-side of the equation to be solved backward from $T$ to 0 .

In the context of the Saint Venant equations, if $\mathbf{W}$ is solution of the backward Saint Venant adjoint equation ( $\mathbf{W}$ has the same structure than $\mathbf{U}$ ), the analysis above leads to:

$$
\int_{(0, T) \times \Omega} j_{\mathbf{U}} \mathbf{U}_{\psi}=-\int_{\Omega} \mathbf{U}_{\psi}(0) \mathbf{W}(0)+\int_{(0, T) \times \Omega} \mathbf{W} S_{\psi}(\mathbf{U}, \psi),
$$

where $S$ is the source term in the Saint Venant equations (3). Indeed, for the Saint Venant equations the direct dependency in $\psi$ is only in $g h \nabla \psi$ in the momentum equations. Also, as in our case there is no direct dependency between the initial condition $\mathbf{U}(0)$ and $\psi$ the first term in the right-hand-side of (23) vanishes. Denoting $\mathbf{W}=\left(w_{1}, \mathbf{w}_{\mathbf{2}}\right)^{t}$ with $\mathbf{w}_{\mathbf{2}}$ the adjoint variable associated 
to $\mathbf{u}$, one has in weak form:

$$
\int_{(0, T) \times \Omega} \mathbf{W} S_{\psi}(\mathbf{U}, \psi)=-\int_{(0, T) \times \Omega} g \nabla \cdot\left(h \mathbf{w}_{\mathbf{2}}\right) .
$$

Slip or Dirichlet boundary conditions give the same homogeneous conditions for the corresponding adjoint variables removing the boundary terms in weak form.

\section{Concluding remarks}

Minimization principles have been used in a context of fluid-structure coupling and the adaptation of a soft structure to a flow in order to minimize some functional under geometric and state constraints. This study, therefore, takes place in the context of shape optimization for unsteady flows where both time dependent and independent shapes can be targeted. Bottom sea motion is an example of the former and building defence structures against erosion the latter. For instance, one can design an immersed structure with geotextile tube [4] to target a flow having a given energy spectrum (e.g. spectrum of waves breaking on the shore based on the number of waves versus their height for a given time interval). When used to model sandy bottom seas evolutions, the study shows that the outcome of the coupling has suitable qualitative behavior comparable to what observed on natural sites. Still, as in any modelling procedure, one needs now an assimilation step for the two parameters of the model ( $\rho$ and $T$ ).

The flow motion is described by the shallow water equations but this can be extended to more sophisticated models including dispersion effects. Also, the approach can obviously be applied to situations where the flow is described by a spectral approach and where time has been removed from the equations using separation of variables in time and space $[15,3,4]$. This is especially interesting if the functional is based on spectral information as well, as mentioned above.

Several assumptions have been analyzed and the corresponding cost functionals described. The approach enables for the introduction of the physical mechanisms responsible for sand motions. Also, geographic and economical 
considerations can be introduced in the functional.

Sensitivity analysis has been then used to locally minimize these functionals. The gradients of these functionals also provide valuable information to identify dominant factors which should therefore receive more attention in the modelling process. It has been shown that when possible the functional should be chosen in order to take advantage of incomplete sensitivity evaluation which makes the cost of sensitivity evaluation negligible and so the minimization procedure. In cases the functional cannot be of the form of (20), one needs to develop an adjoint solver for the state equations. This is the case for the functionals (7), (12) and (13) described in section 4.

Finally, it has been shown that the minimization approach is equivalent to solving an Exner equation for the bottom with an original flux term linking global and local informations. In one dimension in space, this flux term can be derived as non local function involving at a given point the contributions of upwind regions and the contribution increases with the porosity of the bed.

\section{Acknowledgments}

The author would like to thank P. Azerad, F. Marche and F. Bouchette for helpful discussions around this work, especially in the definition of the functionals and the simulation conditions for the basin at Sogreah Grenoble/France. This work is part of ANR project Mathocean (ANR-08-BLAN-0301-01).

The adjoint Saint Venant solver has been obtained using Tapenade AD tool developed at INRIA-Sophia Antipolis by L. Hascoet and his team Tropics http://www-sop.inria.fr/tropics/tapenade.html. 


\section{References}

[1] Audusse, E. Bouchut, F. Bristeau, MO. Klein, R. Perthame, B. (2004). A fast and stable well-balanced scheme with hydrostatic reconstruction for shallow water flows. SIAM J.Sci.Comp. 25/6, 2050-2065.

[2] Audusse, E. Bristeau, M.-O. (2005). A well-balanced positivity preserving second order scheme for shallow water flows on unstructured meshes, J. Comp. Phys. Vol. 206, 311-333.

[3] Azerad, P. Bouchette, F. Isebe, D. Mohammadi, B. (2008). Optimal shape design of coastal structures minimizing short waves impact. Coastal Engineering. Vol. 55/1.

[4] Azerad, P. Bouchette, F. Isebe, D. Mohammadi, B. (2008). Shape optimization of geotextile tubes for sandy beach protection. International Journal for Numerical Methods in Engineering, Vol. 74, 1262-1277.

[5] Barré de Saint Venant A. J.-C. ( 1871). Théorie du mouvement nonpermanent des eaux, avec application aux crues des rivières et à l'introduction des marèes dans leur lit. C.R. Acad. Sc. Paris; Vol. 73, 147154.

[6] Brenier, Y. Benamou, J.D. (2000). A computational fluid mechanics solution to the Monge-Kantarovich mass transfer problem, Numerish Math. 84-3. 375-393.

[7] Dean, R.G. Dalrymple, R.A. (2004). Coastal processes with Engineering Applications, Cambridge University Press.

[8] Dean, R.G. Dalrymple, R.A. (1991). Water Wave Mechanics for Engineers and Scientists, World Scientific Publishing.

[9] Dervieux, A. and Thomasset, F. (1981). Multifuid incompressible fows by a finite element method, Lecture Notes in Physics, Vol. 11, 158-163. 
[10] Eymard, R. Gallouët, T. Herbin, R. (2000). Finite volume methods. In Handbook of numerical analysis, vol VII, Ciarlet PG, Lions JL, editors. North Holland: Amsterdam, 729-1020.

[11] Fowler, A.C. (2001). Dunes and drumlins. Geomorphological fluid mechanics (eds. N.J. Balmforth and A. Provenzale), Springer-Verlag, Berlin, 430454 .

[12] Griewank, A. (2001). Computational derivatives, Springer, New York.

[13] Hasselmann, D.E. Dunckel, M. Ewing, J.A. (1980). Directional Wave Spectra Observed during JONSWAP 1973, Journal of Physical Oceanography, Vol. 10-8. 1264-1280.

[14] Hasselman et al. (1973). Measurements of Wind-Wave Growth and Swell Decay during the Joint North Sea Project (JONSWAP). Ergansungsheft, Reihe A(8), Nr. 12, Deutschen Hydrografischen Zeitschrift.

[15] Kirby, J.T. Dalrymple, R.A. (1995). REFDIF1 v2.5 Refraction diffraction model, Technical report, CACR. Short A.D 1999. Beach and Shoreface Morphodynamics, Wiley.

[16] Kobayashi. N. Desilva, GS. Watson, KD. (1989). Wave transformation and swash oscillation on gentle and steep slopes. J. Geophys. Res. Vol. 94, 951966.

[17] Marche, F. Bonneton, P. (2006). A simple and efficient well-balanced scheme for 2D bore propagation and run-up over a sloping beach. in Proceedings of Int. Conf. Coast. Eng. ICCE, Springer.

[18] Marche, F. Bonneton, P. Fabrie, P. Seguin, N.A. (2002). Evaluation of well-ballanced bore-capturing schemes for $2 \mathrm{D}$ wetting and drying processes, IJNMF.

[19] Mei, C.C. (1989). The applied dynamics of ocean surface waves. World Scientific Publishing. 
[20] Mohammadi, B. Pironneau, O. (2009). Applied shape optimization for fluids, $2^{\text {nd }}$ edition. Oxford Univ. Press.

[21] Mohammadi, B. (2007). Optimal transport, shape optimization and global minimization, C. R. Acad. Sci. Paris, 3511.

[22] Mohammadi, B. Pironneau, O. (2004) Shape Optimization in Fluid Mechanics, Annual Revue of Fluid Mechanics, 36-1.

[23] Mohammadi, B. (2006). Global optimization, level set dynamics, incomplete sensitivity and regularity control, Int. J. CFD, 21-2.

[24] Osher, S. and Sethian, J. (1988). Fronts propagating with curvature-dependent speed: Algorithms based on Hamilton-Jacobi formulations, Journal of Computational Physics, 79(1), 12-49.

[25] Paola, C. Voller, V. R. (2005). A generalized Exner equation for sediment mass balance, J. Geophys. Res., 110.

[26] Peskin, Ch. (1998). The fluid dynamics of heart valves: experimental, theoretical and computational methods, Annu. Rev. Fluid Mech.,Vol. 14, 235259 .

[27] Vilani, C. (2003), Topics in optimal transportation, Graduate Studies in Mathematics series, AMS. 58.

[28] Yang, C.T. (1995). Sediment transport: theory and practice, Mc Graw Hill Education Europe. 

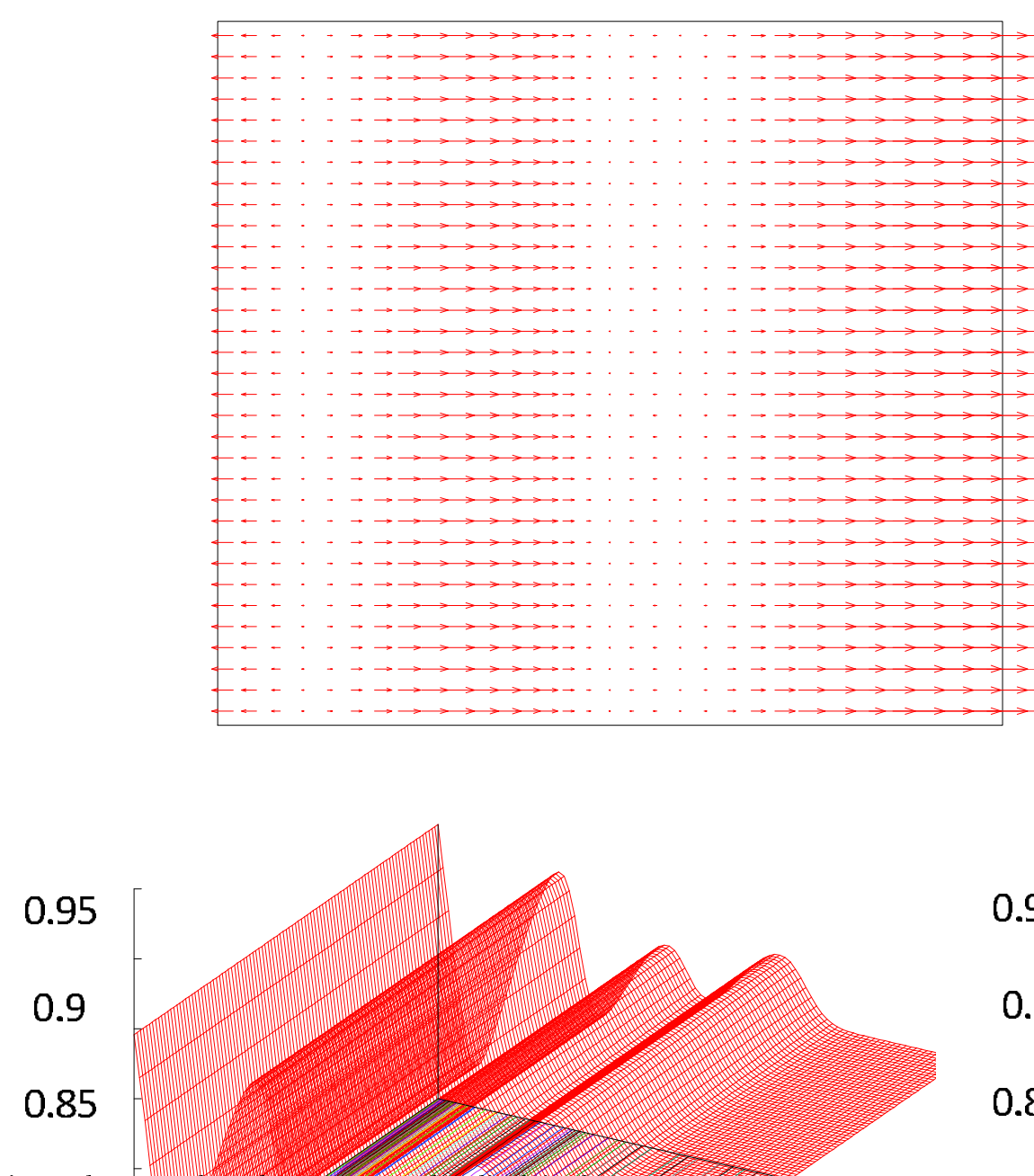

Figure 1: Example of flow in a domain ovithout and with an emerged cylindrical structure modelled by a level set function. Snapshols of Alow velocity fielas (upper line) and water elevation (lower line) for both cases. In particular, one sees the slip boundary condition on tDe Z75locity is well enforced by the level set formulation.

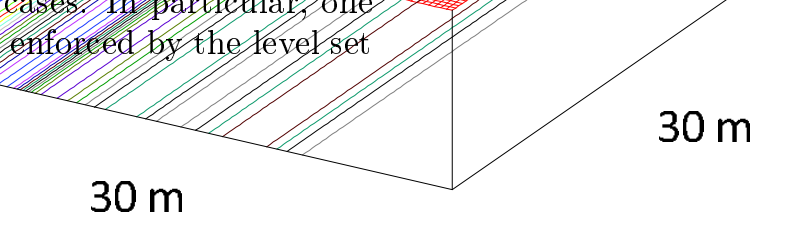



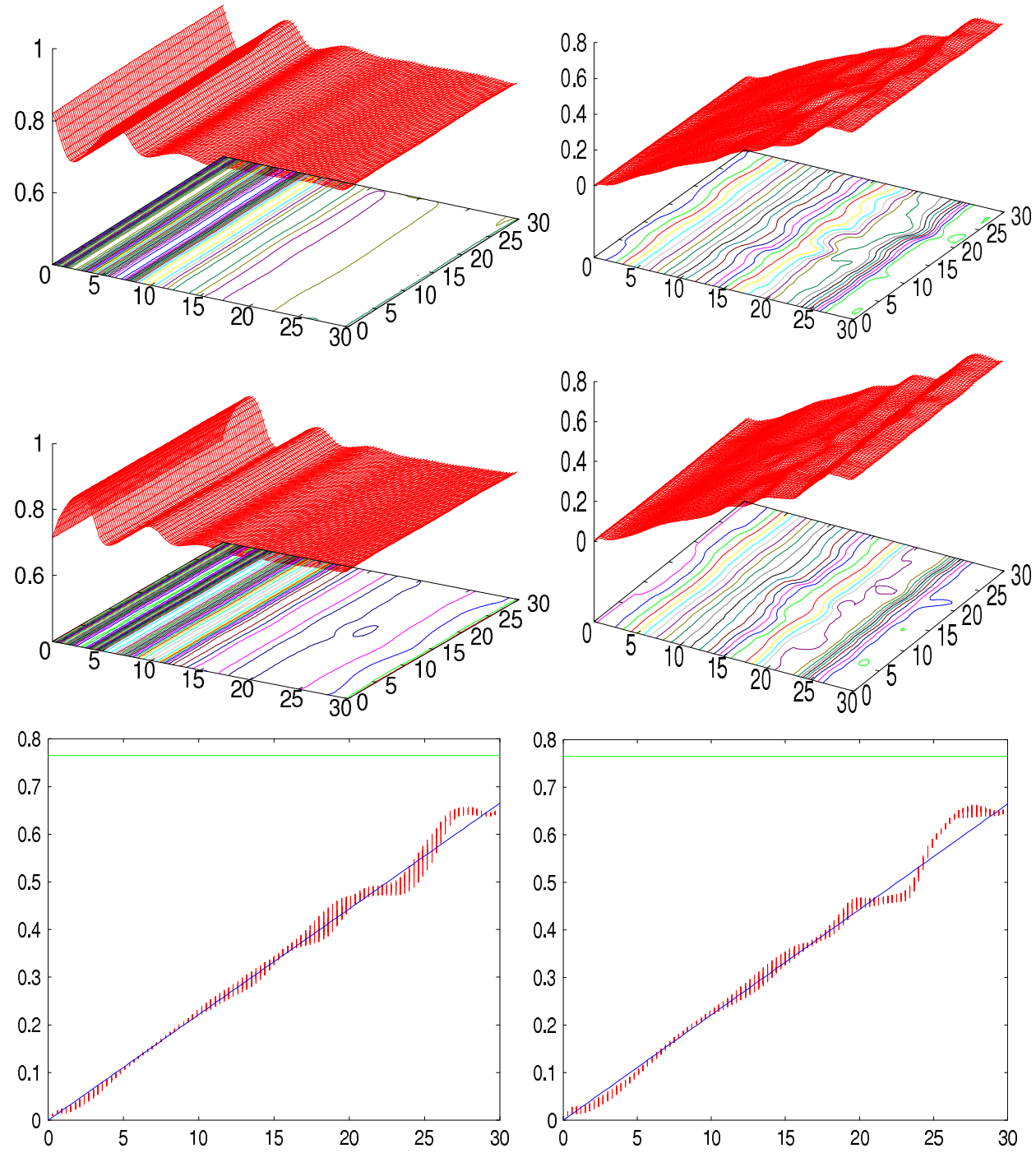

Figure 2: Bottom morphodynamics by fluid-structure coupling based on minimization principles. Upper line: water elevation (left) and bathymetry changes (right) after 10 hours. Middle line: same after 20 hours. Lower line shows bathymetry variabilities longshore. The coupling is started from an initial linear bathymetry. The horizontal line indicates water level at rest. 

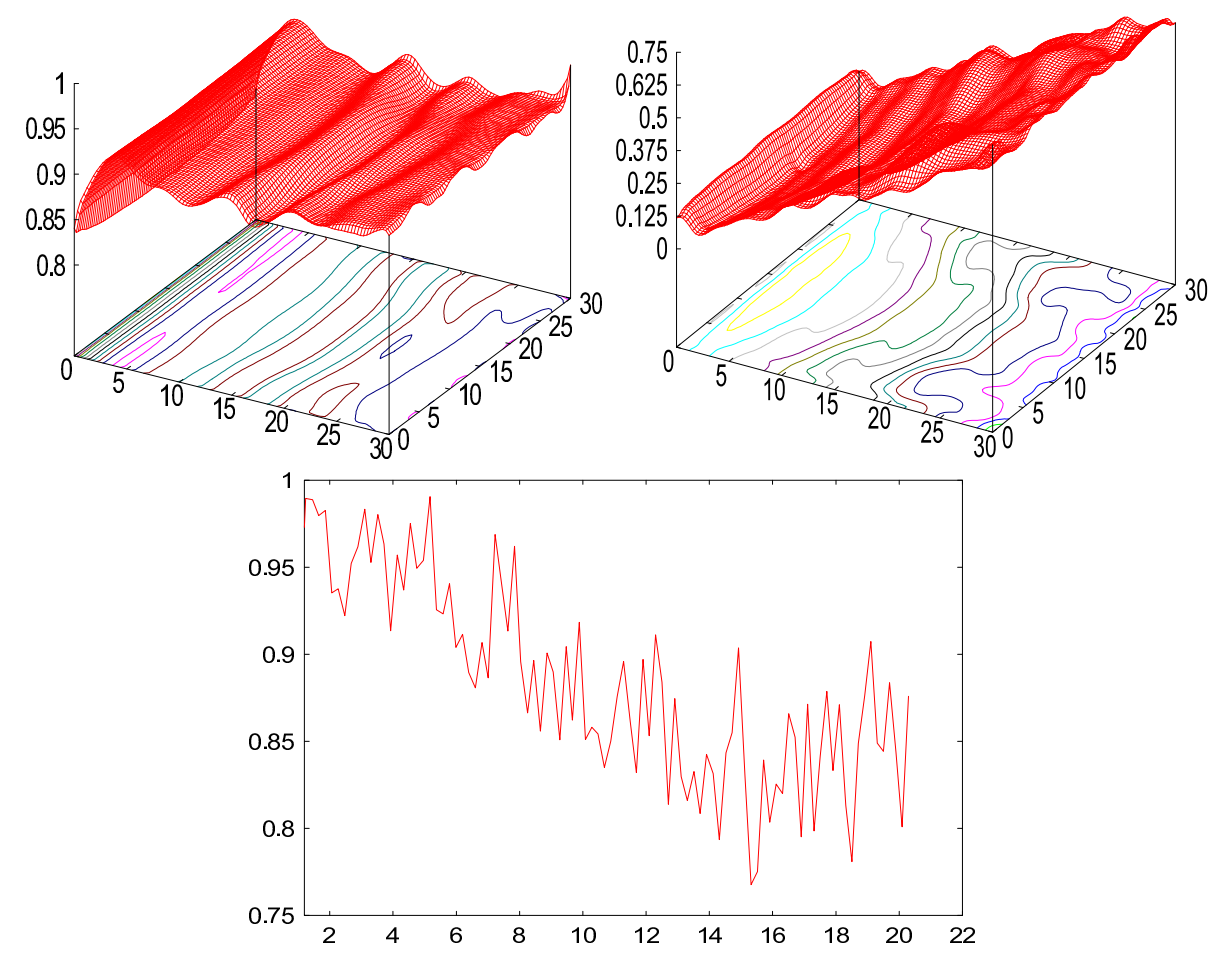

Figure 3: Bottom changes minimizing $J_{2}$ after 20 hours starting from a linear bottom. Water elevation (upper/left), bathymetry (upper/right) and evolution of the normalized cost function in time (lower).
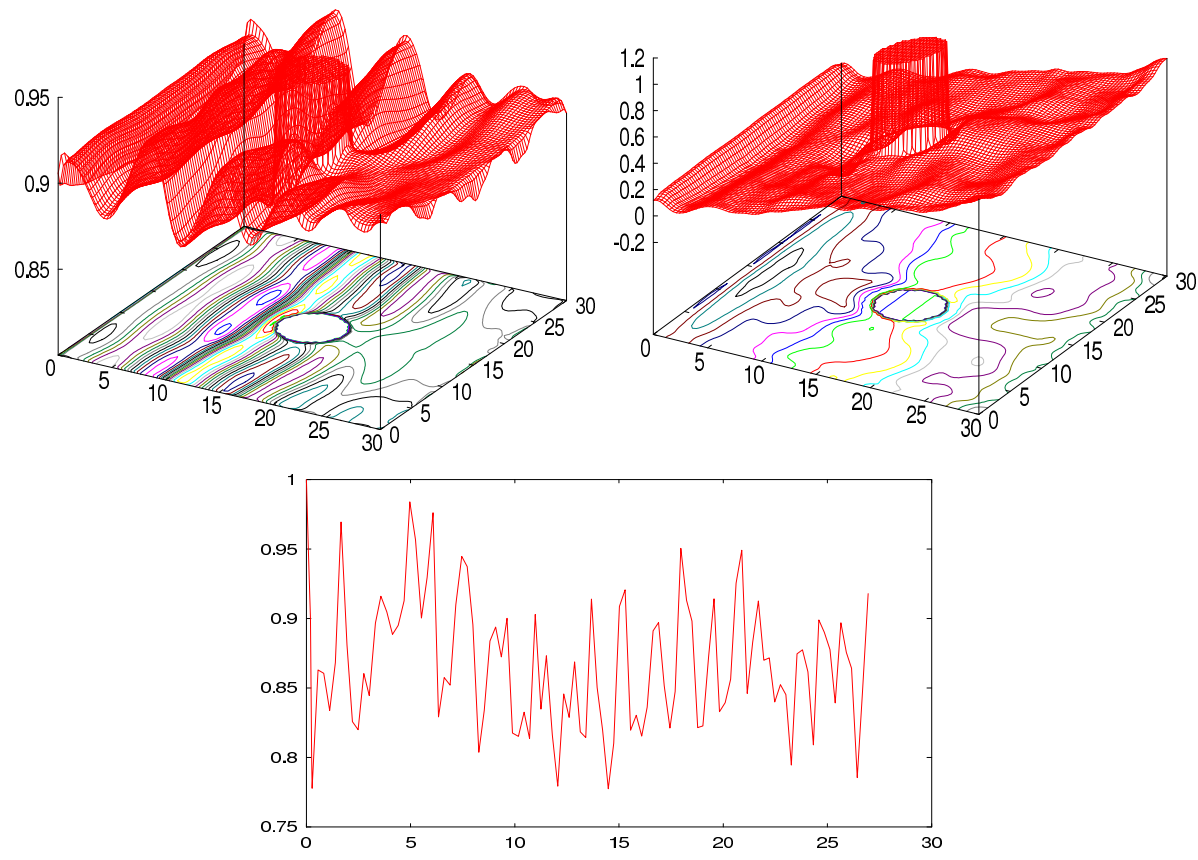

Figure 4: Bottom changes minimizing $J_{2}$ starting from a linear bottom plus an immersed cylindrical structure. Water elevation (upper/left), bathymetry (upper/right) and evolution of the normalized cost function in time (lower). 

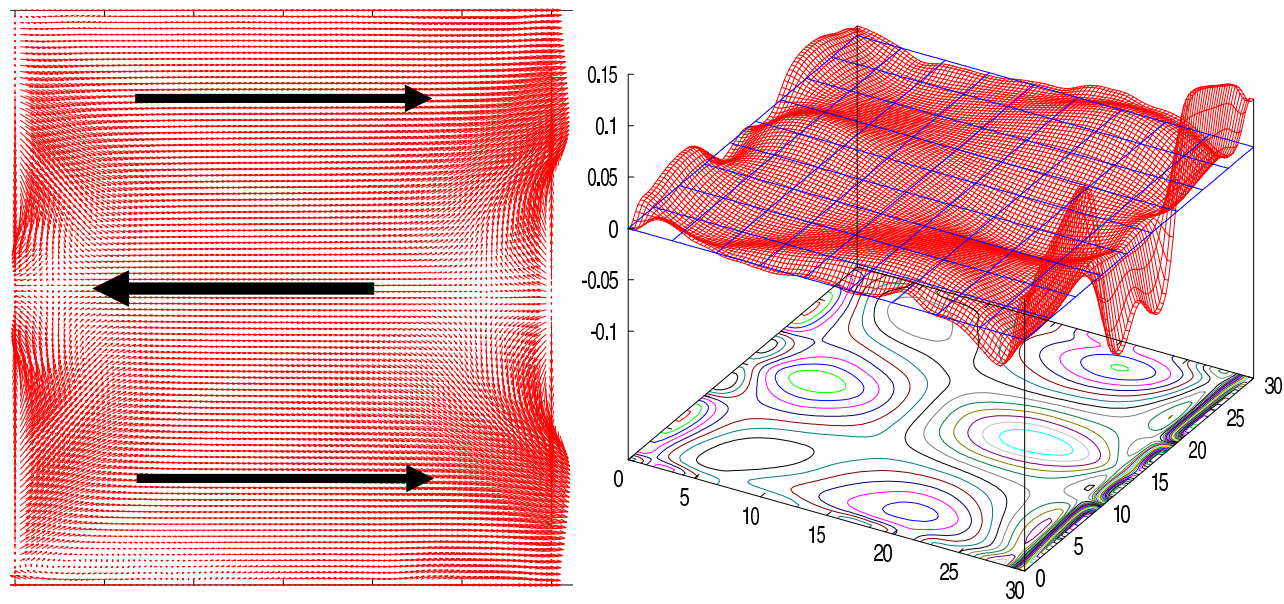

Figure 5: Finding a bottom surface (right) generating a target mean current (left). On the flat bottom the mean current is zero. The flow elevation is periodic and prescribed on the left boundary. The final shape is far from being intuitive. 